Purdue University

Purdue e-Pubs

CTRC Research Publications

Cooling Technologies Research Center

2015

\title{
Near-Field Focusing Sensor for Characterization of Void Content in Thin Dielectric Layers
}

\author{
S. H. Taylor \\ Purdue University \\ S V. Garimella \\ Purdue University, sureshg@purdue.edu
}

Follow this and additional works at: https://docs.lib.purdue.edu/coolingpubs

Taylor, S. H. and Garimella, S V., "Near-Field Focusing Sensor for Characterization of Void Content in Thin Dielectric Layers" (2015). CTRC Research Publications. Paper 246.

http://dx.doi.org/doi:10.1088/0957-0233/26/1/015601

This document has been made available through Purdue e-Pubs, a service of the Purdue University Libraries. Please contact epubs@purdue.edu for additional information. 


\title{
Near-field focusing sensor for characterization of void content in thin dielectric layers
}

\author{
S H Taylor and S V Garimella ${ }^{1}$ \\ Cooling Technologies Research Center, an NSF I/UCRC \\ School of Mechanical Engineering \\ Purdue University \\ West Lafayette, IN 47907 USA
}

\begin{abstract}
A sensor concept is developed and analyzed for in situ characterization of a thin dielectric layer. An array of long, planar electrodes is flush-mounted into opposing faces of two substrates on either side of the dielectric layer. The substrates are oriented such that the lengthwise dimensions of the opposing electrodes are orthogonal. Capacitance is measured between single electrode pairs on opposite substrates while all other electrodes are grounded. The electric field between the active electrodes is sharply focused at their crossing point, resulting in high sensitivity to void content in a square detection zone of the dielectric layer. For a fixed interfacial gap size, direct proportionality of the capacitance with void fraction within the detection zone is poor for high electrode-to-electrode spacing on the substrates, but improves dramatically as this spacing is reduced. Three methods of deriving a simulation-based sensitivity response of measured capacitance to any arbitrary two-dimensional void geometry are investigated. The best method requires data from simulations of an empty air gap and a TIM-filled gap, and uses a reduced-order superposition technique to predict the normalized capacitance value obtained for any void geometry to within $10 \%$ of that predicted by a highly-fidelity direct simulation. The sensing technique is demonstrated using manually introduced voids of $250 \mu \mathrm{m}$ to $2000 \mu \mathrm{m}$ diameter in a $254 \mu \mathrm{m}$-thick interface material layer with a dielectric constant of 4.7. The relationship of the capacitance to the void fraction is shown to fall within the predicted bounds.
\end{abstract}

Keywords: thermal interface, dielectric, capacitance, impedance, nondestructive, void, tomography

1. Corresponding author: sureshg@purdue.edu 
Sensor for characterization of void content in dielectric layers

Submitted to Measurement Science and Technology

\section{Introduction}

Dielectric materials are ubiquitous in industrial processes and products. Dielectric thermal interface materials (TIMs) are often used in electronic packages, where efficient thermal transport is essential to continuous function. Morphology characterization of these materials is required for predicting performance. Measurement techniques used to characterize these materials may be used for other media, such as polymer sheets or dielectric fluids in narrow microfluidic channels, where nonintrusive void/bubble detection is desired. In microelectronic packages, heat generated by the processor must transfer through multiple intermediate components before it can be dissipated to the ambient. Thermal interface materials serve to minimize thermal contact resistances at component interfaces and prevent overheating. Without a TIM layer present, heat flow is constricted to the solid-spot contacts between asperities on mating hard surfaces, which may only offer $1-2 \%$ of the nominal contact area [1].

Predictive modeling of the time-dependent thermal conductance of TIM layers in service conditions informs industrial development of high-performance electronics [2]. Analytical and semiempirical models of thermal interface contact resistances have been reviewed by Prasher [3] and Gwinn and Webb [4]. Void formation is the primary thermal performance limitation for TIM layers. The effects of TIM-to-substrate interface resistances may be considered when estimating the total layer resistance [3], but these are often inconsequential compared to the resistance due to voids in the bulk material. The influence of voids on the TIM layer thermal resistance may be readily simulated by considering a reduced thermal conductivity within the void region. Ankireddi and Copeland [5] simulated thermal transport in a large set of TIM layers with randomly distributed voids to produce statistical performance metrics that inform quality control guidelines for package assembly.

The uniformity of a TIM layer is typically determined by assembly processes and thermomechanical stresses experienced in service, and therefore, experimental characterization approaches that evaluate the evolution of performance in situ, during the course of thermomechanical 
Sensor for characterization of void content in dielectric layers

cycling, are required for obtaining real-time feedback on the TIM design. Methods for experimental evaluation of TIM layers include direct evaluation of thermal performance $[1,4,6,7]$, indirect void detection by infrared imaging [8,9] or infrared microscopy [10], and physical characterization. Firstorder physical characterization of voids may either be performed nondestructively with transparent substrates which cannot mimic TIM-substrate thermomechanical interactions experienced in real applications, or by destructive investigation which only provides data at a single point in time [7]. More detailed, nondestructive physical characterization of TIM layers may be achieved with acoustic microscopy $[7,9,11]$ or x-ray imaging [11].

The present work develops a new technique for the physical characterization of electrically insulating layers by capacitance measurement. Impedance-based sensing has found applications in the measurement or detection of liquid level [12, 13], displacement [14], pressure [15, 16], pH level [17, 18], chemical concentration [18], polyimide curing [19], coating degradation [20, 21], flow regime [22], phase distribution in multi-phase flow over a surface [23], and imaging of buried objects [24]. Capacitancebased sensing may be similarly applied for void detection in dielectric TIM layers. Capacitance is measured using electrodes embedded in the mating substrates. The contrast in dielectric strength between the TIM material and the void regions provides a means for void detection. Ultimately, the method could provide a means to monitor an experimental TIM layer in situ as the material develops void regions, providing high-temporal-resolution data over aging or thermal cycling processes without pausing the thermal schedule of the material under test.

One convenient electrode configuration for capacitance-based sensing is to place an array of long, parallel electrodes on either side of the interfacial gap, oriented orthogonal to each other (orthogonal mesh configuration); this provides a grid of crossing points at which capacitance may be measured. Immersed wire-mesh sensors employing this configuration ( 0.1 mm electrode diameter with 2-3 mm pitch) have been used to characterize a cross-section of multiphase flow [25], with a binarized capacitance measured at each crossing-point corresponding to the local fluid phase. This approach has also been demonstrated for packed beds [26] with resolution corresponding to the electrode pitch. As an extension of this 
Sensor for characterization of void content in dielectric layers

concept, a field-focusing sensor has been used to characterize the phase distribution in flow through a channel between parallel plates containing embedded electrodes [27]. The resolution of this sensor degrades rapidly with increasing channel height, as the region of strong electrical field created by actuating an opposing electrode pair begins to significantly overlap with corresponding regions between adjacent pairs. Although phase maps obtained from the field-focusing configuration may be improved with a deconvolution algorithm [28], spatial overlap in electric fields created by different pairs, as well as soft-field effects, obfuscate the task of assigning a local void fraction to each measurement.

An orthogonal mesh configuration of opposing substrate-embedded electrodes is analyzed here for an interfacial gap filled with a TIM layer as a means of obtaining a set of local void fractions over finite sections of the TIM footprint. Three reduced-order models for characterizing the sensitivity response of a representative crossing point (junction) are evaluated against direct simulation of a large set of random 2D void geometries. The reduced-order models are capable of providing an estimate of local void fraction in the TIM layer, to within uncertainty bounds that depend on sensor design. An experimental apparatus is used to demonstrate sensor detection of voids that are between $250 \mu \mathrm{m}$ and $2000 \mu \mathrm{m}$ in diameter present in a dielectric TIM layer of $254 \mu \mathrm{m}$ thickness.

\section{Sensor description}

The near-field focusing sensor consists of two substrates containing electrodes flush-mounted into opposing substrate surfaces as shown in figure 1. The electrode surfaces are exposed to the TIM layer in between the substrates. Capacitance measurements taken between electrode pairs, at junctions where they overlap, yield information regarding the effective two-dimensional distribution of permittivity of the layer. When the gap is filled with a dielectric medium, any air voids present near a junction reduce capacitance. It has been shown that, for an electrode width of $640 \mu \mathrm{m}$, femto-Farad measurement resolution is sufficient to resolve partial voiding of a junction region in a $25 \mu \mathrm{m}$ dielectric layer [29]. 
Sensor for characterization of void content in dielectric layers

In the current work, a $254 \mu \mathrm{m}$-thick dielectric solid-phase TIM layer is considered. An experimental test unit, with electrode width $w=640 \mu \mathrm{m}$, electrode depth $d=850 \mu \mathrm{m}$, and electrode pitch $p=1550 \mu \mathrm{m}$ (see figure 1) is used for validation of the sensing approach. The test cell contains two arrays of five copper electrodes each, embedded in transparent acrylic substrates. Plastic spacers fit between the substrates to create a $254 \mu \mathrm{m}$ gap. The TIM material chosen for simulation and experimental validation is Tflex SF210, with an estimated dielectric constant of 4.7 at the measurement frequency [30].

An electrical characterization station was created with individual grounding probes for each of the electrode contacts and manually operated excitation and receiver probes. The excitation and receiver probes are shielded by a coaxial grounded sheath. To eliminate parasitic capacitance between the excitation and receiver probes, the probe length beyond shielding is minimized $(\sim 1 \mathrm{~mm})$. Capacitance is measured at $32 \mathrm{kHz}$ with a commercial meter (Analog Devices, AD 7746) with absolute uncertainty of \pm 4 $\mathrm{fF}$ [31]. Repeatability variation for several cases of a non-voided TIM layer was $\pm 2 \mathrm{fF}$.

Junction capacitances in the sensor array are measured sequentially for individual single opposing-electrode pairs, with the inactive electrodes held at the virtual ground of the capacitance meter. One electrode of the active pair serves as the excitation electrode, oscillating between ground and the supply voltage $V_{\mathrm{DD}}$, while the other electrode serves as the receiver, held at $V_{\mathrm{DD}} / 2$. Measurement of the desired capacitance with an oscillating driving potential in this stray-immune configuration [32] neutralizes spurious capacitance paths leading from the receiver to any constant-potential body such as nearby grounded electrodes. Charge transfer from the excitation to the receiver determines the effective capacitance of the pair. When a dielectric TIM layer fills the gap between substrates, the junction capacitance is higher than for an air gap; measured capacitance values that fall between the known values for TIM-filled and air gaps indicate a partially-voided condition in the TIM layer in the region of the junction. 


\section{Electric field modeling}

The capacitance measurements in this work are taken at low frequency $(32 \mathrm{kHz})$, resulting in a quasistatic electric field in the active electrode junction region of interest. Electric potential, $\varphi$, is distributed throughout the domain according to the divergence of the electric displacement field,

$$
\nabla \cdot(\varepsilon \nabla \varphi)=0
$$

For the active junction, the top electrode acts as the excitation electrode, oscillating between supply voltage, $\varphi_{\mathrm{exc}}=V_{\mathrm{DD}}$, and virtual ground, $\varphi_{\mathrm{exc}}=0$, while the bottom electrode acts as receiver electrode, held constant at $\varphi_{\mathrm{exc}}=V_{\mathrm{DD}} / 2$. During the excitation wave trough, electric flux is from the receiver electrode to the excitation electrode, as shown in figure 2. At the crest, the electric field is reversed with the flux going from the excitation to the receiver electrodes, but remains largely unchanged between the receiver electrode and grounded neighboring electrodes. The capacitance of the junction is determined by the net charge transfer seen by the receiver electrode, $\Delta Q$.

For modeling purposes, the charge transfer to the receiver electrode may be calculated as the surface integral of the net displacement electric field on the exposed faces of the receiving electrode,

$$
\Delta Q=\left(\int \varepsilon \nabla \varphi \cdot \mathrm{d} \mathbf{S}\right)_{\text {crest }}-\left(\int \varepsilon \nabla \varphi \cdot \mathrm{d} \mathbf{S}\right)_{\text {trough }}
$$

Experimentally, the discharge is calculated via current $I$ into the receiver electrode during the step change as

$$
\Delta Q=\int_{t}^{t+\Delta t} I \mathrm{~d} \tau
$$

The junction capacitance is calculated as

$$
C=\frac{\Delta Q}{V_{D D}} .
$$

The simulation domain and boundary conditions for a representative internal sensor junction are shown in figure 3. Two electrostatic numerical simulations are performed with the excitation electrode 
Sensor for characterization of void content in dielectric layers

set at $\varphi_{\mathrm{exc}}=0$ and $\varphi_{\mathrm{exc}}=V_{\mathrm{DD}}$, with $V_{\mathrm{DD}}=1 \mathrm{~V}$. The charge transfer is calculated according to (2) using the simulation results at both excitation conditions.

In the simulation, a second-order 7-point numerical scheme is used to solve (1). A regular mesh of approximately 400,000 control volumes is used. The harmonic average of neighboring-cell permittivity values is used in the finite-difference spatial derivative approximations between adjacent cells. Due to the high $L / H$ aspect ratio of the layer, the limiting factor in grid independence is the number of cells bridging the thickness, $H$. Eight control volumes were found sufficient to resolve the gradient across the gap, with a junction capacitance change of less than $0.03 \%$ when doubling the gap resolution. The TIM material, acrylic substrate, and air are modeled with dielectric constants of 4.7 [30], 3.0 [33], and 1.0 respectively.

\section{Reduced-order sensitivity function models}

\subsection{Two-dimensional approximation}

Capacitance values obtained from the simulation for the solid-TIM and air gap cases are $214 \mathrm{fF}$ and $65 \mathrm{fF}$, respectively. The span between these two values provides the means for detecting partial voiding conditions when the measurement resolution is on the order of $1 \mathrm{fF}$. The detection volume of the junction is defined as the $p \times p \times H$ gap volume centered at the junction. In this analysis, the void fraction characterization is simplified by approximating the gap region as two-dimensional. Thus, all voids are simulated as having constant cross-section in the $z$ dimension, and the reduced-order model analysis is performed on the two-dimensional detection zone, $\Omega$, constituting the $p \times p$ region centered at the crossing point. A sensitivity function $\psi(x, y)$ defined on the detection zone is proposed to describe the spatial sensitivity of the measured capacitance to any arbitrary void geometry, $\omega$, as shown in figure 4 .

Three different methods of deriving such a sensitivity function are explored. In this analysis, capacitance is normalized as

$$
C^{*}=\frac{C_{S}-C}{C_{S}-C_{V}},
$$


Sensor for characterization of void content in dielectric layers

where $C_{S}$ is the capacitance of the junction when filled with the TIM, and $C_{V}$ is the capacitance of the junction as an air gap. The normalized capacitance of the junction, $C^{*}$, follows the junction region void fraction, $\chi$, from 0 at no voiding $(\chi=0)$ to 1 at complete voiding $(\chi=1)$. In the subsequent analysis, the detection zone is also normalized by the electrode width, $p$, to a unit square, and the sensitivity function, $\psi(x, y)$, is normalized such that $C^{*}$ is obtained through integration of $\psi(x, y)$ on the unit square. Thus, the forward problem of obtaining a predicted measurement given an arbitrary void geometry is simplified from a $3 \mathrm{D}$ electric field simulation to a $2 \mathrm{D}$ integral evaluation. In the following subsections, three proposed methods of deriving the function $\psi(x, y)$ are developed, and their accuracy evaluated when directly used to obtain $C^{*}$ for random void geometries.

\subsection{Method 1: Perturbation}

The perturbation method of characterizing sensitivity of junction capacitance exhaustively catalogues the response of a small void placed in each of an array of possible locations throughout the domain. The magnitude of the change in capacitance of the junction is taken as the junction sensitivity at the $x-y$ coordinate in the center of the void. A set of simulations is used to characterize the sensitivity $\psi_{1}(x, y)$ for the detection zone, using a $61 \mu \mathrm{m} \times 61 \mu \mathrm{m}$ square void placed sequentially in a $25 \times 25$ array of locations within a one-quarter symmetry portion of the detection zone. The sensitivity function is normalized such that

$$
1=\int \psi_{1}(x, y) \mathrm{d} \Omega
$$

The sensitivity function for the perturbation characterization method is shown in figure 5. The capacitance measurement is most sensitive to the central $w \times w$ region where the electrodes cross, and least sensitive in the 'dead regions' at the corners of the detection zone (low values of $\psi_{1}$ ). The sensitivity function may be used to estimate the normalized capacitance for any void distribution as

$$
C^{*}=\int \psi_{1}(x, y) \mathrm{d} \omega
$$


Sensor for characterization of void content in dielectric layers

\subsection{Method 2: Voxel Capacitance}

The voxel capacitance method requires division of the domain into a set of voxels, and allocates sensitivity according to the capacitance of each voxel, as may be derived from the energy, $U$, stored in a capacitor

$$
U=\frac{1}{2} \Delta V^{2} C
$$

where $\Delta V$ is the voltage difference across the electrodes. Combined with the volume integral of energy in an electric field, the capacitance is expressed as

$$
C=\frac{1}{\Delta V^{2}} \int \varepsilon|\mathbf{E}|^{2} \mathrm{~d} v
$$

A discretization of this integral may be used to approximate the capacitance of each voxel, $C_{\mathrm{n}}$, as

$$
C_{\mathrm{n}} \cong \frac{1}{\Delta V^{2}} \varepsilon_{\mathrm{n}}\left|\mathbf{E}_{\mathrm{n}}\right|^{2} v_{\mathrm{n}}
$$

When implementing this method, the cells of the mesh are used as the voxels. Equation (10) is used to assign a capacitance value to every voxel after calculating the distribution of electric field, $\mathbf{E}$, from the numerical solution of electric potential, $\varphi$, according to

$$
\mathbf{E}=-\nabla \varphi
$$

The sensitivity function may be calculated from just the solutions of the solid TIM case and the complete void case. To reduce the dimensionality of the sensitivity function, $z$-direction dependence is incorporated through summation to provide an integral-averaged value of the sensitivity at the $x-y$ location. The $x-y$ distribution of capacitance on the regular mesh may be expressed as

$$
C_{\mathrm{i}, \mathrm{j}}=\frac{\varepsilon}{V_{\mathrm{DD}}^{2}} \sum_{\mathrm{n}=1}^{\mathrm{k}} v_{\mathrm{i}, \mathrm{j}, \mathrm{n}}\left|\mathbf{E}_{\mathrm{i}, \mathrm{j}, \mathrm{n}}\right|^{2}
$$

where all voxels within the $p \times p \times H$ detection volume are considered, and $\mathrm{i}, \mathrm{j}$, and $\mathrm{k}$ index according to the coordinate directions. Equation (12) is a discrete function that can be used to represent the sensitivity over the detection zone for the solid TIM, $\psi_{2, \mathrm{~S}}(x, y)$, and complete void, $\psi_{2, \mathrm{v}}(x, y)$, cases, shown in figure 6. 
The sensitivity functions are scaled such that the volume under the surface is equal to unity. Any arbitrary void geometry may be modeled using a superposition of these two cases as,

$$
C^{*}=1-\frac{1}{\frac{C_{S}}{C_{V}}-1} \int\left(\frac{C_{S}}{C_{V}} \psi_{S}-\psi_{V}\right) \mathrm{d} \omega .
$$

\subsection{Method 3: Mid-plane Flux}

The third method considers the electric displacement field at the mid-plane of the gap between substrates to characterize the two-dimensional junction sensitivity. Conservation of electric displacement flux requires that the net flux through any $z$ plane in the detection zone must equal the net flux into the receiver electrode (neglecting interference from nearby grounded electrodes). The distribution of displacement flux, $\boldsymbol{\varepsilon} \mathbf{E}_{\mathrm{m}}$, normal to the mid-plane of the detection zone may be represented as

$$
\varepsilon \mathbf{E}_{\mathbf{m}}=\left(\left(\left.\varepsilon \nabla \varphi\right|_{\mathrm{x}, \mathrm{y}, \frac{\mathrm{H}}{2}}\right)_{\text {crest }}-\left(\left.\varepsilon \nabla \varphi\right|_{\mathrm{x}, \mathrm{y}, \frac{\mathrm{H}}{2}}\right)_{\text {trough }}\right) \cdot \mathbf{k} .
$$

The capacitance of the junction may be written as

$$
C=\frac{1}{V_{D D}} \int \varepsilon \mathbf{E}_{\mathbf{m}} \mathrm{d} \Omega
$$

The magnitude of $\varepsilon \mathbf{E}_{\mathrm{m}}$ serves as a two-dimensional sensitivity function for the detection zone. Like the voxel capacitance method, this method requires only two simulations to characterize a known gap thickness, $H$. The sensitivity function is normalized such that the volume under the surface is equal to unity for both the solid TIM case, $\psi_{3, \mathrm{~S}}(x, y)$, and the complete void case, $\psi_{3, \mathrm{~V}}(x, y)$, as shown in figure 7 . Normalized capacitance, $C^{*}$, is calculated by (13). The mid-plane flux method provides a smoother sensitivity function than the perturbation or voxel methods.

\subsection{Assessment of sensitivity derivation methods}

The three sensitivity functions account for reduction of the capacitance through attenuation of the electric displacement field by voiding; however, all three functions are subject to two sources of error: the 
Sensor for characterization of void content in dielectric layers

assumption of complete independence of detection zones, and the presence of 'soft-field' effects near the permittivity step-change of the void perimeter/TIM interface.

The first source of error arises from the influence on the capacitance measurement from voiding outside the detection zone. The inactive electrodes are grounded to minimize this effect by damping the electric field beyond the active junction; however, the capacitance of the junction will still exhibit some dependency on the void distribution in adjacent detection zones. The normalized error, $E^{*}$, obtained by using a sensitivity function is defined by

$$
E^{*}=\frac{C_{\psi}-C}{C_{S}-C_{V}}
$$

where $C_{\psi}$ indicates the value obtained through use of the sensitivity function, and $C$ indicates the simulated capacitance value. The normalized error represents the positive or negative error in predicted capacitance as a proportion of the range between the air gap and TIM-filled cases.

Error associated with the influence of voids outside the active detection zone may be quantified by comparing direct simulations of capacitance to values obtained from a sensitivity function for worstcase scenarios. The error of the mid-plane flux capacitance prediction for a TIM-filled detection zone when the adjacent detection zones are air-filled is illustrated in table 1(b) and visa versa in table 1(e). For these unlikely cases, normalized errors of 0.093 and -0.126 are observed. A less conservative scenario featuring circular void geometries of diameter $\mathrm{p}$ centered in adjacent zones is also shown, with errors of 0.072 (table 1(c)) and -0.093 (table 1(f)).

The soft-field effects arise from the distortion of electric field pathlines due to nonuniform permittivity distributions. Soft-field effects increase in proportion to the dielectric contrast $\left(\varepsilon_{\mathrm{TIM}} / \varepsilon_{\mathrm{Void}}\right)$ [32]. Here, isolated soft-field effects are not analyzed, but a statistical study is conducted, as described below, to quantify the overall error resulting from both sources.

To gauge relative accuracy of the three methods, a Monte Carlo approach with 500 cases is used to produce a statistical comparison of the capacitance response obtained by the reduced-order sensitivity functions versus direct simulation. Each case consists of a simulated junction containing between one and 
Sensor for characterization of void content in dielectric layers

five elliptical void regions of random location, size, and orientation. Elliptical axes of the voids vary randomly in size between $0.02 p$ and $1.6 p$. The union of all voids constitutes the region, $\omega$, for each case. Random cases were generated until a selected set of 500 could uniformly span all void fractions in $10 \%$ increments. Select cases are illustrated in figure 8.

Each case is simulated directly to establish the exact capacitance value, $C$, and then compared to the capacitance value obtained through each of the three sensitivity function approximations. A box plot of error distribution for each method is shown in figure 9 with statistics provided in table 2. Each of the methods underpredicts the direct simulation by several percent. The mid-plane flux method performs best with a normalized mean error of -0.021 and standard deviation of 0.020 as shown in table 2 . The errors are attributed to soft-field effects and codependency of adjacent detection zones.

The set of cases analyzed suggests a weak dependence of error on void fraction, with high void fraction cases generally displaying larger underestimates. However, a detailed study of error as a function of void fraction, including soft-field effects and the bias introduced by the condition of neighbor zones (see table 1) is beyond the scope of the current work.

\section{Inverse procedure for error quantification}

While data obtained from the sensor are insufficient for reconstructing exact void geometries, they may be used to obtain a set of localized void fractions corresponding to the set of $p \times p$ regions composing the domain of interest. In this section, the sensitivity function derived using the mid-plane flux method is used to solve the inverse problem of quantifying the potential error of using a normalized capacitance measurement as a direct indicator of void fraction. The utility of the near-field focusing sensor lies in the prediction of void fraction in a single detection zone by the normalized capacitance,

$$
\chi \approx C^{*}
$$

When (17) is used to estimate an unknown void fraction from a capacitance measurement, some level of error is introduced because it is not known whether the void is located in the central region of high 
Sensor for characterization of void content in dielectric layers

sensitivity in the detection zone, or in a peripheral region of low sensitivity. The sensitivity function may be used to bound this error by considering two limiting cases of void distribution, as demonstrated in figure 10(a) using the mid-plane flux method. In the first case, voiding begins at the most sensitive central region of the detection zone, and grows by preferring the next most sensitive available region of the detection zone. In this case, normalized capacitance increases sharply, and provides an overestimate of void fraction, as shown by the upper curve in figure 10(a). In the second case, voiding begins at the least sensitive peripheral region of the detection zone, and grows by preferring the next least sensitive available region. In this case, normalized capacitance lags behind void fraction, providing an underestimate, as shown by the bottom curve. The curves create a bounding envelope that quantifies the potential error in estimating void fraction as the normalized capacitance value in (17). This error is due to the dead regions in the corners of the detection zone, which result from wide electrode spacing. Because the two bounding curves were created from the mid-plane flux sensitivity function, each is subject to the error, $E^{*}$, associated with that sensitivity function (table 2). The total potential error of (17) is $E^{*}$ in addition to the error bounds depicted in figure 10(a).

As can be seen from figure $\mathbf{1 0}$ (a), errors as large as $\pm 20 \%$ can occur when normalized capacitance is used to estimate void fraction for the simulated geometry where there is a large spacing between electrodes $(p / w=2.42)$; however, agreement between void fraction and normalized capacitance improves as the spacing between electrodes decreases, as seen in figure 10(b). In the limit $(p / w \rightarrow 1)$, dead regions no longer exist, and the envelope collapses, representing independence of void morphology (albeit still subject to the error, $E^{*}$, of the sensitivity function).

\section{Experimental results}

Experimental values of $C_{S}$ and $C_{V}$ are roughly $20 \%$ higher than the simulation values as shown in table 3. In order to calibrate for this discrepancy, experimental values of $C_{S}$ and $C_{V}$ are used in (13). As the simulation represents an internal junction surrounded by grounded electrodes, the sensitivity function shown in figure 7 applies only to the $3 \times 3$ set of internal junctions of the system. 
Sensor for characterization of void content in dielectric layers

In the experiment, 17 different cases were tested using Tflex SF210. Through voids of different diameters and locations were created in $1 \mathrm{~cm} \times 1 \mathrm{~cm}$ samples of the TIM. The sample and spacers were first placed on one substrate, and then the opposing substrate was pressed on top. A fixed weight was used to apply a constant pressure of $12 \mathrm{kPa}$ in all the tests.

In the test cases, artificial voids were created ranging between $250 \mu \mathrm{m}$ and $2000 \mu \mathrm{m}$ in diameter. All voids were placed within the detection zones of the $3 \times 3$ set of internal junctions. The void outlines were measured optically by viewing the void through the transparent substrates with a $5 \mathrm{X}$ magnification microscope. Void shapes were approximated as 2D, and fitted to an ellipse. Uncertainty in the phase boundaries due to $z$-direction variations near the void edges was quantified by defining outlines corresponding to the minimum and maximum possible void areas as shown in figure 11. Voids in the images were located spatially by observing coordinates of nearby intersections of electrode edges. Repeatability error in the measurements of $C^{*}$ was 0.02 .

Because some voids bridge more than one detection zone, the 17 test cases yield 41 partiallyvoided junctions. Select cases are shown in figure 12, which illustrates the optically identified bounds of void geometry and the capacitance measurements, normalized as $C^{*}$ values.

The experimental capacitance values are compared against those predicted from the mid-plane flux method in figure 13. Each data point is shown as a span between a high and a low estimate, which respectively correspond to the minimum and maximum estimated area outlines of the void. Slight delamination of the TIM (failure to remain in contact with the top substrate) is observed as an annular region surrounding the induced void. This region is included in the estimate of the maximum void size. The data indicate that for cases with a large span in figure 13, this generally represents an overly conservative estimate (bottom of range).

Normalized capacitance as a predictor of void fraction is shown for the data in figure 14, with overlaid solid lines indicating the bounding envelope as predicted using the mid-plane flux method. The data validate the sensor concept as a viable method for estimating void content in the dielectric layer. The 
Sensor for characterization of void content in dielectric layers

smallest void successfully detected in the TIM layer in one detection volume $(1.55 \mathrm{~mm} \times 1.55 \mathrm{~mm} \times 254$ $\mu \mathrm{m}$ ) is approximately $250 \mu \mathrm{m}$ in size, corresponding to a void fraction of roughly 0.02 .

\section{Conclusion}

The near-field focusing sensor with an orthogonal electrode mesh configuration has been demonstrated as a means to measure void content in a thin dielectric layer. Characterization of sensor response with a two-dimensional sensitivity function is described as a means of bounding the error of void fraction estimates obtained with the sensor. Void detection in a $254 \mu \mathrm{m}$ thick thermal interface material is experimentally illustrated using the method developed.

The sensor requires no moving parts, and measurements may be taken continuously. The in situ nature of this method makes it an ideal candidate for monitoring temporally evolving void structures in dielectric layers in real time without stopping or suspending the sample stress schedule for data collection. The simplicity of the instrumentation required makes this technique viable for high-volume testing in an industrial setting.

\section{Acknowledgements}

The authors gratefully recognize financial support for this work from the Cooling Technologies Research Center, a National Science Foundation Industry/University Cooperative Research Center at Purdue University, as well as technical discussions with Dr. Justin Weibel. 
Sensor for characterization of void content in dielectric layers

\section{References}

[1] Singhal V, Litke P J, Black A F and Garimella S V 2005 An experimentally validated thermomechanical model for the prediction of thermal contact conductance International Journal of Heat and Mass Transfer 48 5446-59.

[2] Garimella, S V et al. 2008 Thermal challenges in next-generation electronic systems IEEE

Transactions on Components and Packaging Technologies 31(4) 801-15.

[3] Prasher, R 2006 Thermal interface materials: historical perspective, status, and future directions Proceedings of the IEEE 94(8) 1571-86.

[4] Gwinn, J P and Webb R L 2003 Performance and testing of thermal interface materials Microelectronics Journal 34 215-22.

[5] Ankireddi S and Copeland D 2007 A statistical approach for characterizing the thermal impact of TIM Voids Proc. IEEE Semiconductor Thermal Measurement and Management Symp. (San Jose, CA: IEEE) pp 79-82.

[6] Goel N et al. 2008 Technical review of characterization methods for thermal interface materials Electronics Packaging Technology Conf. Proc. (Singapore: IEEE) pp. 1461-71.

[7] Islam N, Lee S W, Lee J Y, Ka Y H, Khim J Y and Galloway J 2010 TIM selection methodology for high power flip chip packages Intersociety Conf. on Thermal and Thermomechanical Phenomena in Electrical Systems (ITHERM) (Las Vegas, NV: IEEE).

[8] Gupta A, Liu Y, Zamora N and Paddock T 2006 Thermal imaging for detecting thermal interface issues in assembly and reliability stressing Proc. $10^{\text {th }}$ Intersociety Conference on Thermal and Thermomechanical Phenomenon in Electronics Systems (San Diego, CA: IEEE vol 1-2) pp 942-5.

[9] Gowda A, Esler D and Tonapi S 2004 Voids in thermal interface material layers and their effect on thermal performance $6^{\text {th }}$ Electronics Packaging Technology Conf. Proc. (Singapore: IEEE) pp 41-46. [10] Hu X, Jiang L and Goodson K E 2004 Thermal characterization of eutectic alloy thermal interface materials with void-like inclusions $20^{\text {th }}$ Annual IEEE Semiconductor Thermal Measurement and Management Symposium, Proc. 2004 (San Jose, CA: IEEE) pp 98-103. 
Sensor for characterization of void content in dielectric layers

[11] Gowda A, Esler D, Tonapi S, Zhong A, Srihari K and Schattenmann F 2006 Micron and submicron-scale characterization of interfaces in thermal interface material systems Journal of Electronic Packaging 128 130-6.

[12] Shi T M, Xie C G, Huang S M, Williams R A and Beck M S 1991 Capacitance-based instrumentation for multi-interface level measurement Measurement Science and Technology 2 923-33.

[13] Canbolat H 2009 A novel level measurement technique using three capacitive sensors for liquids IEEE Transactions on Instrumentation and Measurement 58(10) 3762-8.

[14] Lei H, Chen X, Yao Y and Li X 2012 Novel quartz crystal capacitive sensor for micro displacement detection IEEE Sensors Journal 12(6) 2145-9.

[15] Zhou M X, Huang Q A, Qin M and Zhou W 2005 A novel capacitance pressure sensor based on sandwich structures Journal of Microelectromechanical Systems 14(6) 1272-82.

[16] Damghanian M and Majlis B Y 2009 Novel design and fabrication of high sensitivity MEMS capacitive sensor array for fingerprint imaging Advanced Materials Research 74 239-42.

[17] Schoning M J, Nather N, Auger V, Poghossian A and Koudelka-Hep M 2005 Miniaturised flowthrough cell with integrated capacitive EIS sensor fabricated at wafer level using Si and SU-8 technologies Sensors and Actuators B 108 986-92.

[18] Schoning M J, Thust M, Muller-Veggian M, Kordos P and Luth H 1998 A novel silicon-based sensor array with capacitive EIS structures Sensors and Actuators B 47 225-30.

[19] Pan Z, Hesketh P J, Maclay G J 1993 Novel embedded capacitive sensors for monitoring the cure of polymide in multichip modules Journal of Vacuum Science and Technology A 11(4) 1396-1400.

[20] Wang X, Mei J and Xiao P 2000 Non-destructive evaluation of thermal barrier coatings using impedance spectroscopy Journal of the European Ceramic Society 21 855-59.

[21] Ogawa K, Minkov D, Shoji T, Sato M and Hashimoto H 1999 NDE of degradation of thermal barrier coating by means of impedance spectroscopy Non-Destructive Testing and Evaluation (NDT\&E) International 32 177-85. 
Sensor for characterization of void content in dielectric layers

[22] Paranjape S, Ritchey S N and Garimella S V 2012 Electrical impedance-based void fraction measurement and flow regime identification in microchannel flows under adiabatic conditions International Journal of Multiphase Flow 42 175-83.

[23] Thiele S, Da Silva M J and Hampel U 2009 Capacitance planar array sensor for fast multiphase flow imaging IEEE Sensors Journal 9(5) 533-40.

[24] Schlicker D, Washabaugh A, Shay I and Goldfine N 2006 Inductive and capacitive array imaging of buried objects Insight 48(5) 302-6.

[25] Da Silva M J, Schleicher E and Hampel U 2007 Capacitance wire-mesh sensor for fast measurement of phase fraction distributions Measurement Science and Technology 18 2245-51.

[26] Schubert M, Kryk H and Hampel U 2010 Slow-mode gas/liquid-induced periodic hydrodynamics in trickling packed beds derived from direct measurement of cross-sectional distributed local capacitances Chemical Engineering and Processing 49 1107-21.

[27] Da Silva M J and Hampel U 2009 A field-focusing imaging sensor for fast visualization of multiphase flows Measurement Science and Technology 20(10) 104009.

[28] Cui Z, Wang H, Chen Z and Yang W 2011 Image reconstruction for field-focusing capacitance imaging Measurement Science and Technology 22(3) 035501.

[29] Taylor S H and Garimella S V 2013 Void detection in dielectric films using a floating network of substrate-embedded electrodes Journal of Electronic Packaging 136041008.

[30] Laird Technologies Inc. 2013 Tflex SF200 series thermal gap filler Data Sheet http://www.lairdtech.com/NewsItem.aspx ?id=2147486923\#.UqnZjNF3sbw.

[31] Analog Devices Inc. 2005 24-bit capacitance-to-digital converter with temperature sensor: AD7745/AD7746 Data Sheet, Norwood, MA.

[32] Hu X and Yang W 2010 Planar capacitive sensors - designs and applications Sensor Review 30(1) 24-39.

[33] TDI International Inc. 2014 Acrylic data sheet Data Sheet http://www.tdiinternational.com/contents/en-us/d597_acrylic-data-sheet.html. 
Sensor for characterization of void content in dielectric layers

\section{List of tables}

Table 1. Simulation results for errors obtained by neglecting void geometries outside the detection zone for different void distributions. Darkened regions indicate voids.

Table 2. Error incurred with the three reduced-order sensitivity methods compared to direct simulation.

Table 3. Experimental values for solid TIM junction capacitance, $C_{S}$, and complete air-void capacitance, $C_{V}$. Corresponding simulation values of a representative junction are $C_{S}=214 \mathrm{fF}, C_{V}=65 \mathrm{fF}$ with $C_{S} / C_{V}$ $=3.26$. Data represents the $3 \times 3$ set of interior junctions. 
Table 1. Simulation results for errors obtained by neglecting void geometries outside the detection zone for different void distributions. Darkened regions indicate voids.

\begin{tabular}{|c|c|c|c|}
\hline Description & $\begin{array}{c}\text { Void } \\
\text { Geometry }\end{array}$ & $\begin{array}{c}\text { Capacitance } \\
C(\mathrm{fF})\end{array}$ & $\begin{array}{c}\text { Normalized } \\
\text { Error } E^{*}\end{array}$ \\
\hline $\begin{array}{l}\text { (a) TIM- } \\
\text { filled in } \\
\text { zone and in } \\
\text { adjacent } \\
\text { zones }\end{array}$ & $p$ & 214 & 0 \\
\hline $\begin{array}{l}\text { (b) Voided } \\
\text { in adjacent } \\
\text { zones }\end{array}$ & & 200 & 0.093 \\
\hline $\begin{array}{l}\text { (c) Voids of } \\
\text { size } p \\
\text { centered in } \\
\text { adjacent } \\
\text { zones }\end{array}$ & & 203 & 0.072 \\
\hline $\begin{array}{l}\text { (d) Voided } \\
\text { in zone and } \\
\text { in adjacent } \\
\text { zones }\end{array}$ & & 65 & 0 \\
\hline $\begin{array}{l}\text { (e) TIM- } \\
\text { filled } \\
\text { adjacent } \\
\text { zones }\end{array}$ & & 84 & -0.126 \\
\hline $\begin{array}{l}\text { (f) TIM } \\
\text { regions of } \\
\text { size } p \text { in } \\
\text { adjacent } \\
\text { zones }\end{array}$ & & 79 & -0.093 \\
\hline
\end{tabular}


Sensor for characterization of void content in dielectric layers

Table 2. Error incurred with the three reduced-order sensitivity methods compared to direct simulation.

\begin{tabular}{lcc}
\hline \multicolumn{1}{c}{ Method } & $E^{*}$ Mean & $E^{*}$ Std. Dev. \\
\hline 1. Perturbation & -0.024 & 0.025 \\
2. Voxel Capacitance & -0.029 & 0.047 \\
3. Mid-plane Flux & -0.021 & 0.020 \\
\hline
\end{tabular}


Sensor for characterization of void content in dielectric layers

Table 3. Experimental values for solid TIM junction capacitance, $C_{S}$, and complete air-void capacitance, $C_{V}$. Corresponding simulation values of a representative junction are $C_{S}=214 \mathrm{fF}, C_{V}=65 \mathrm{fF}$ with $C_{S} / C_{V}$ $=3.26$. Data represents the $3 \times 3$ set of interior junctions.

\begin{tabular}{|c|c|c|}
\hline \multicolumn{3}{|c|}{ Solid TIM: $C_{S}(\mathrm{fF})$} \\
\hline 272 & 266 & 272 \\
\hline 270 & 264 & 270 \\
\hline 273 & 267 & 274 \\
\hline \multicolumn{3}{|c|}{ Complete Void: $C_{V}(\mathrm{fF})$} \\
\hline 82 & 77 & 83 \\
\hline 82 & 78 & 84 \\
\hline 85 & 82 & 88 \\
\hline \multicolumn{3}{|c|}{ Ratio: $C_{S} / C_{V}$} \\
\hline 3.32 & 3.45 & 3.28 \\
\hline 3.29 & 3.38 & 3.21 \\
\hline 3.21 & 3.26 & 3.11 \\
\hline
\end{tabular}




\section{Table of Figures}

Figure 1. Schematic diagram of near-field focusing sensor.

Figure 2. Two-dimensional illustration of junction cross-section boundary conditions at the trough and crest of the excitation signal during capacitance measurement with $V D D=1$. Dashed line indicates 2D projection of simulation domain.

Figure 3. Simulation domain for a single internal junction, extending to grounded sidewalls of adjacent electrodes.

Figure 4. Top view of simulation domain with $p \times p$ detection zone and void sub-domain.

Figure 5. Normalized junction sensitivity function in the detection zone calculated by the perturbation characterization method.

Figure 6. Normalized junction sensitivity function in the detection zone calculated from the voxel capacitance method for a solid TIM case (a) and completely voided case (b).

Figure 7. Normalized junction sensitivity function in the detection zone calculated from the mid-plane flux method for a solid TIM case (a) and completely voided case (b).

Figure 8. Selected cases from the set of 500 random void distributions used to compare the capacitance response obtained by reduced-order methods versus direct simulation. The $p \times p$ detection zones are shown, with black regions indicating voids.

Figure 9. Error distributions of reduced-order sensitivity characterization methods, calculated from the set of 500 random cases of void distribution.

Figure 10. (a) Void fraction prediction envelope obtained by the mid-plane flux method (inset graphics represent void fractions of 0.3 and 0.7 ) and (b) variation of this envelope for decreasing ratios of electrode pitch to width.

Figure 11. Photograph of void in TIM layer as viewed through the transparent substrate.

Figure 12. Experimental void geometries for selected cases with black and gray respectively indicating the optically measured small estimate and large estimate of void geometry, (left), with experimentally measured values of $C^{*}$ on the $3 \times 3$ grid of electrode junctions (right). Measurements of $\left|C^{*}\right|<0.02$ are eliminated as noise. Case (c) corresponds to figure 11 .

Figure 13. Junction capacitance due to the presence of artificial voids as measured experimentally and predicted using the visualized void boundaries. Each prediction is shown as a span between small and large void boundary limits. 
Sensor for characterization of void content in dielectric layers

Figure 14. Optically measured void fraction versus normalized capacitance overlaid with void fraction bounds obtained from the mid-plane flux method. 
Sensor for characterization of void content in dielectric layers

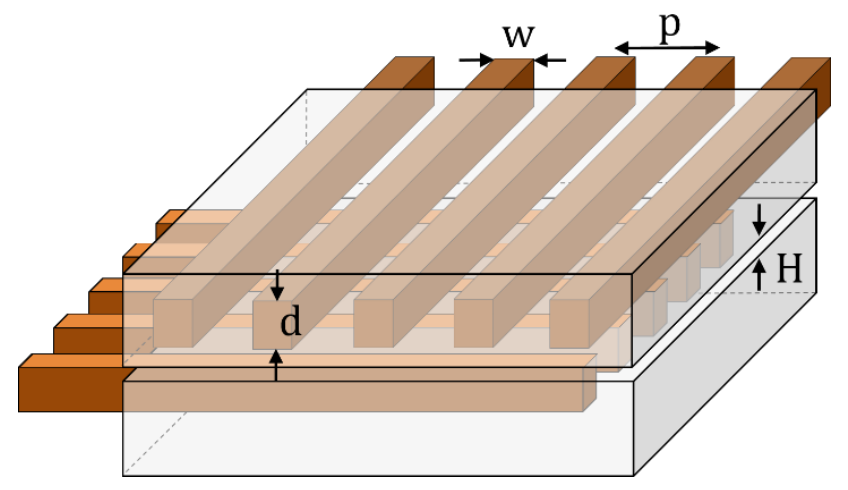

Figure 1. Schematic diagram of near-field focusing sensor. 
Sensor for characterization of void content in dielectric layers
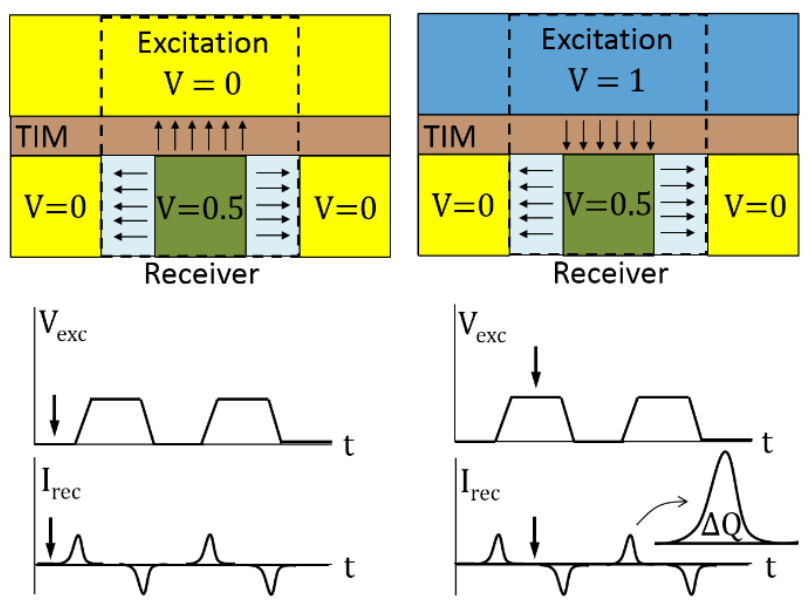

Figure 2. Two-dimensional illustration of junction cross-section boundary conditions at the trough and crest of the excitation signal during capacitance measurement with $V_{\mathrm{DD}}=1$. Dashed line indicates 2D projection of simulation domain. 
Sensor for characterization of void content in dielectric layers

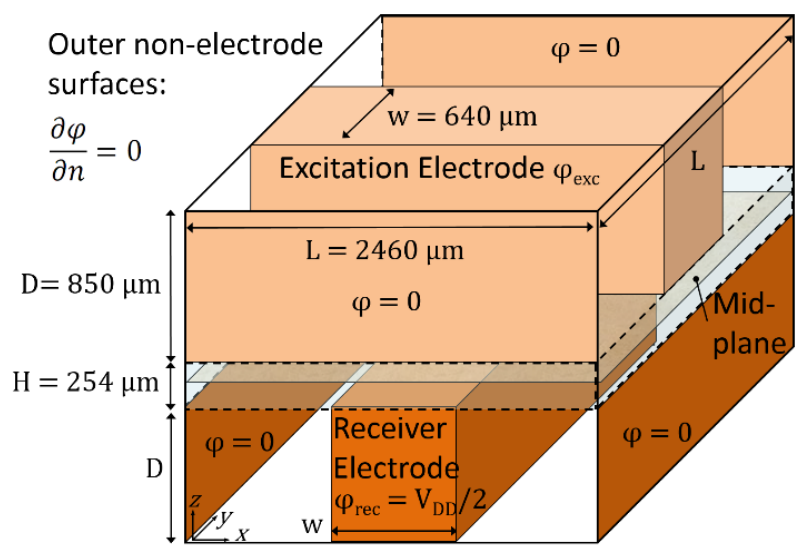

Figure 3. Simulation domain for a single internal junction, extending to grounded sidewalls of adjacent electrodes. 
Sensor for characterization of void content in dielectric layers

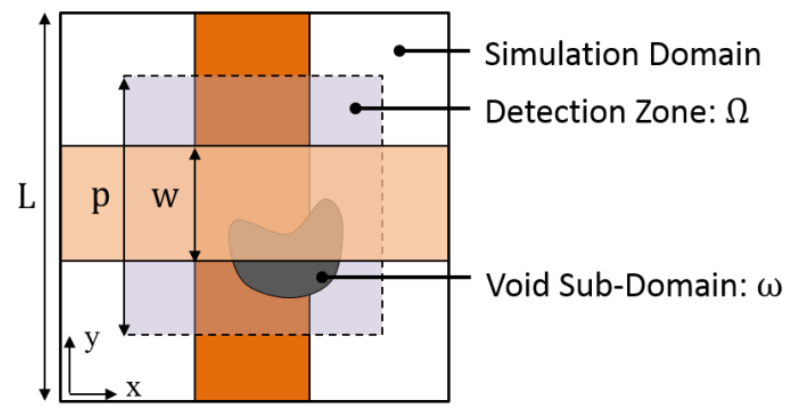

Figure 4. Top view of simulation domain with $p \times p$ detection zone and void sub-domain. 
Sensor for characterization of void content in dielectric layers

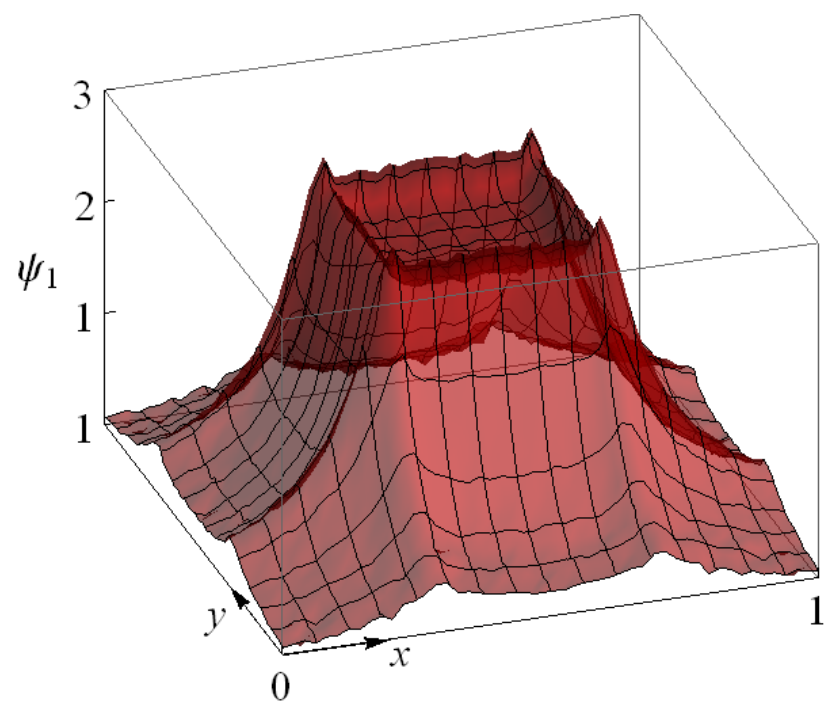

Figure 5. Normalized junction sensitivity function in the detection zone calculated by the perturbation characterization method. 
Sensor for characterization of void content in dielectric layers

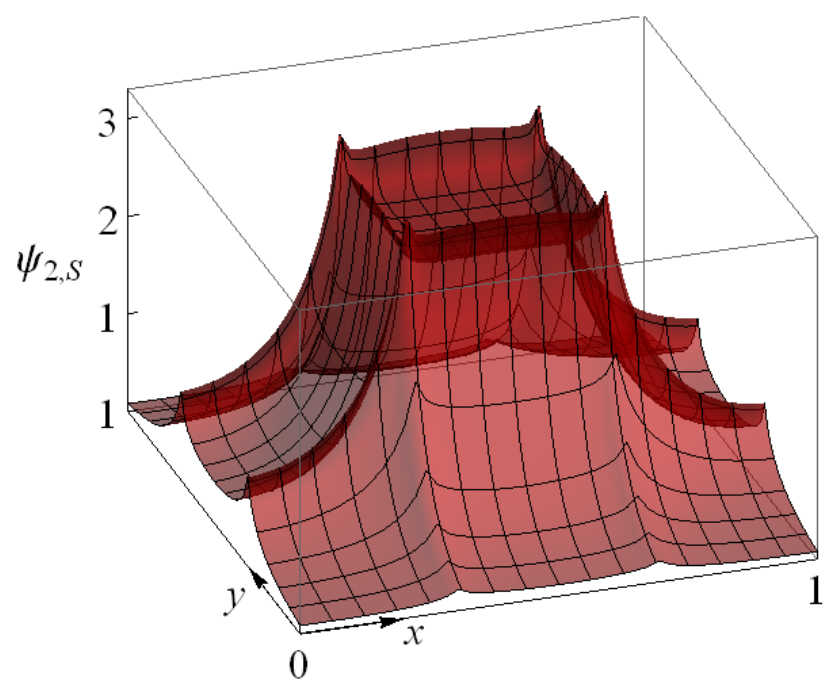

(a)

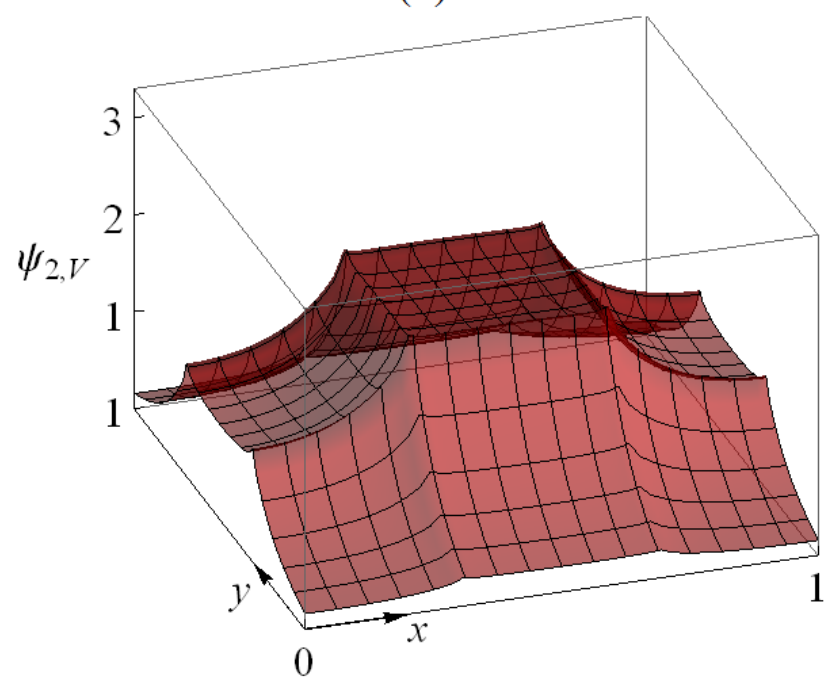

(b)

Figure 6. Normalized junction sensitivity function in the detection zone calculated from the voxel capacitance method for a solid TIM case (a) and completely voided case (b). 
Sensor for characterization of void content in dielectric layers

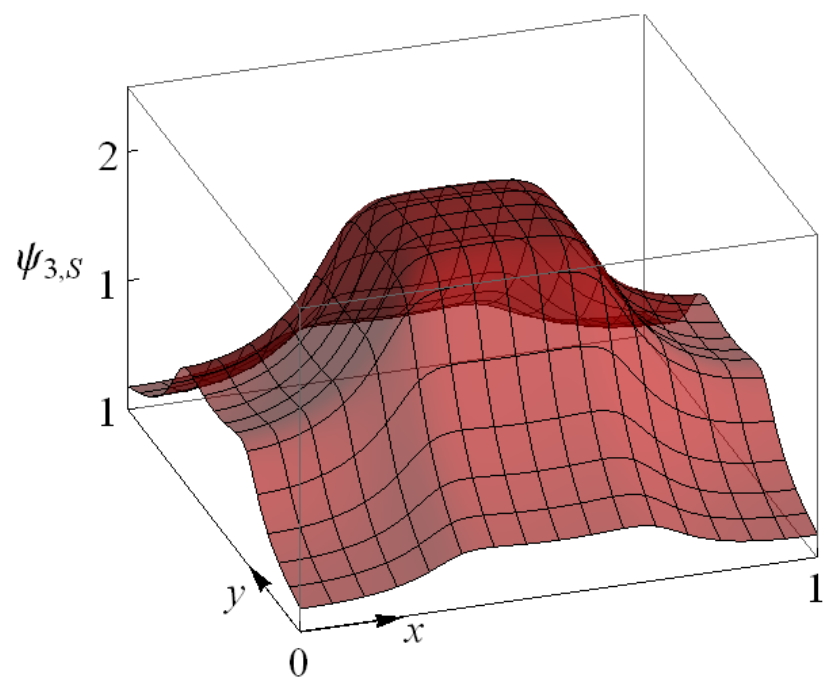

(a)

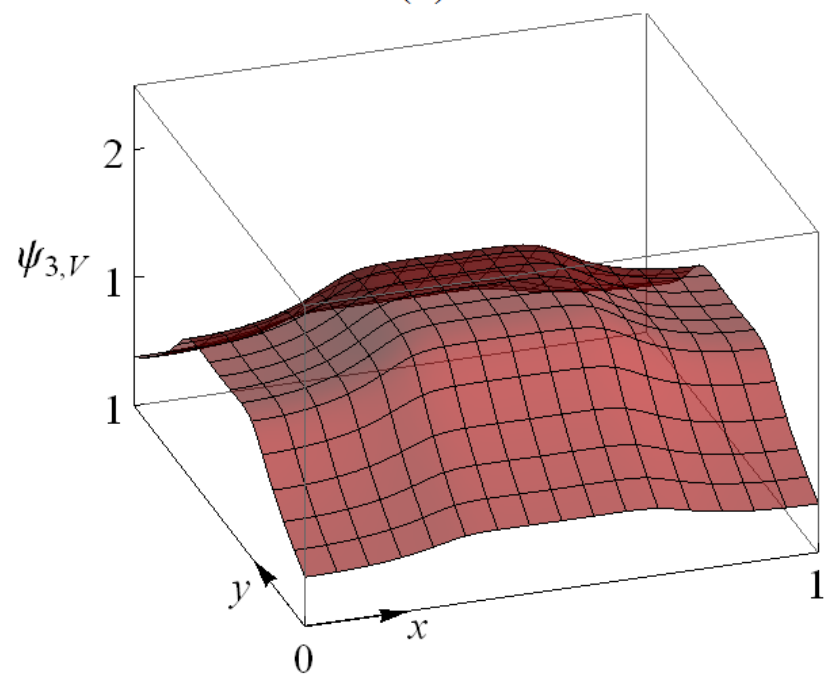

(b)

Figure 7. Normalized junction sensitivity function in the detection zone calculated from the mid-plane flux method for a solid TIM case (a) and completely voided case (b). 


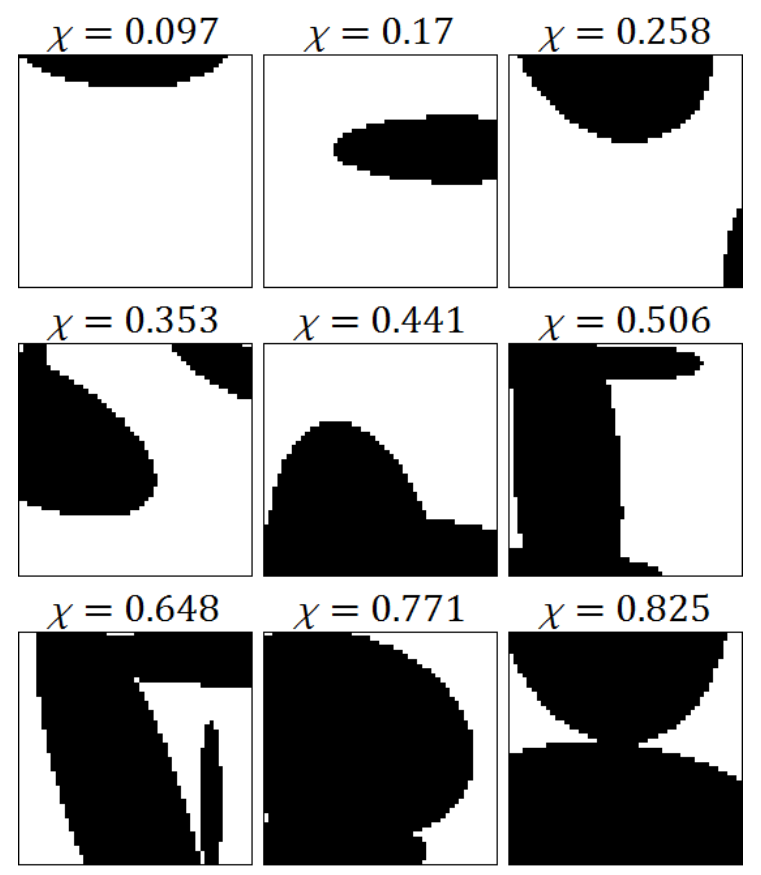

Figure 8. Selected cases from the set of 500 random void distributions used to compare the capacitance response obtained by reduced-order methods versus direct simulation. The $p \times p$ detection zones are shown, with black regions indicating voids. 
Sensor for characterization of void content in dielectric layers

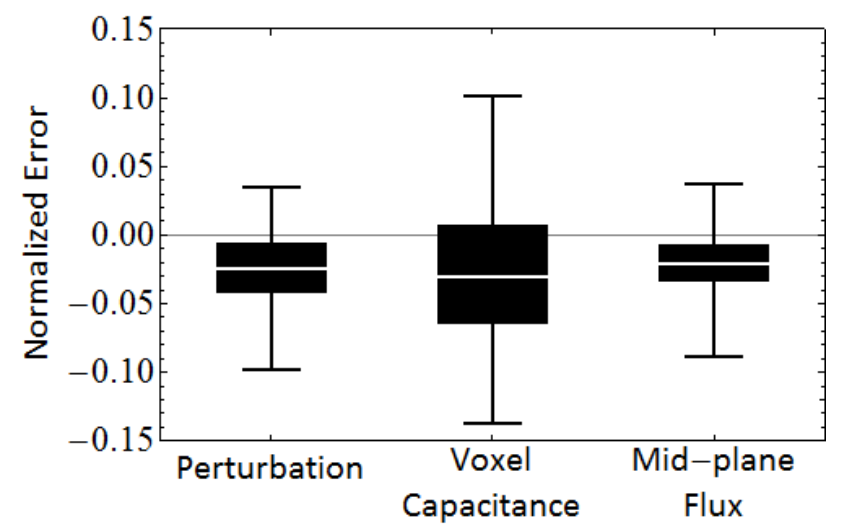

Figure 9. Error distributions of reduced-order sensitivity characterization methods, calculated from the set of 500 random cases of void distribution. 
Sensor for characterization of void content in dielectric layers

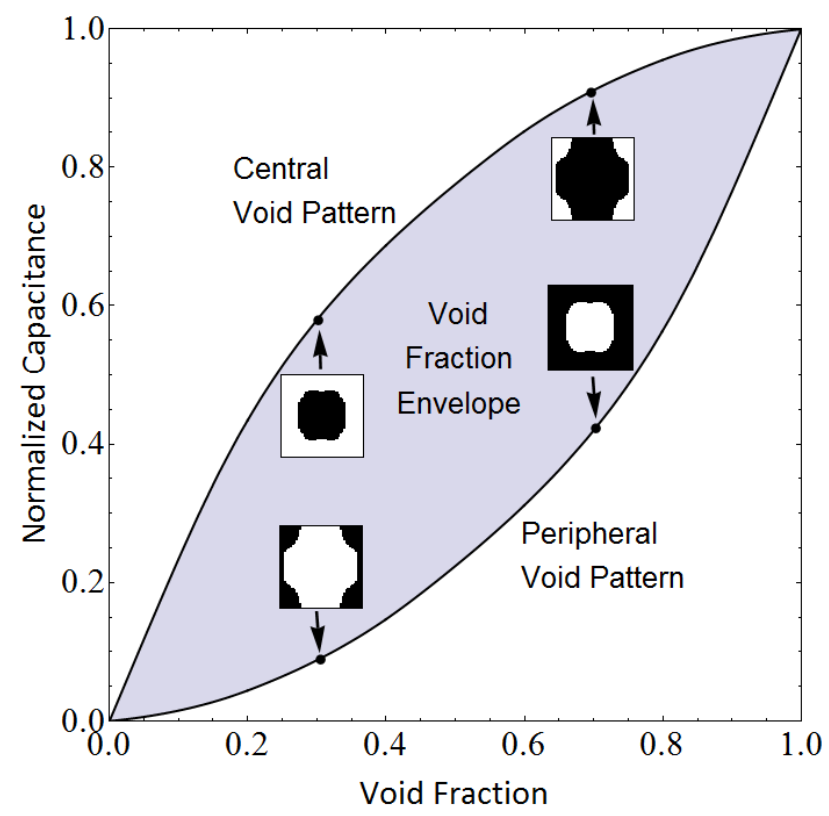

(a)

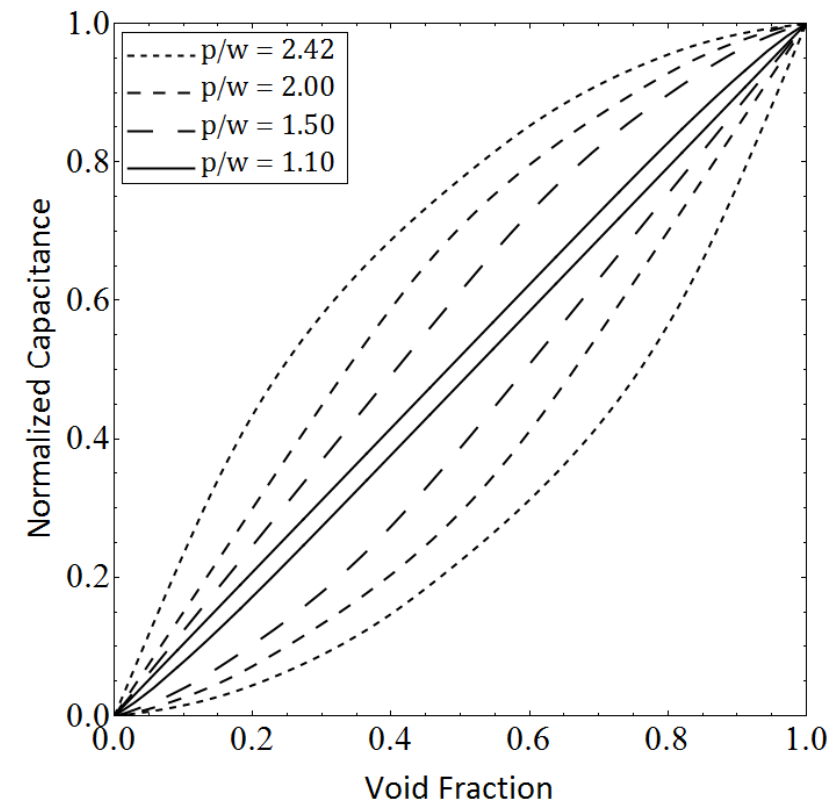

(b)

Figure 10. (a) Void fraction prediction envelope obtained by the mid-plane flux method (inset graphics represent void fractions of 0.3 and 0.7 ) and (b) variation of this envelope for decreasing ratios of electrode pitch to width. 
Sensor for characterization of void content in dielectric layers

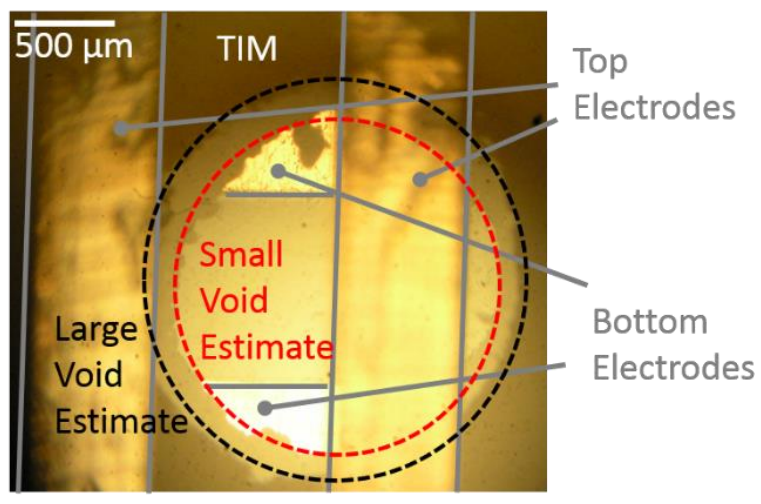

Figure 11. Photograph of void in TIM layer as viewed through the transparent substrate. 
Sensor for characterization of void content in dielectric layers

(a)

\begin{tabular}{|l||l||l||l|l|l|}
\hline \multicolumn{1}{|c||}{} & & & 0 & 0 & 0 \\
\hline \hline & & & 0 & 0.14 & 0 \\
\hline \hline & & & 0 & 0 & 0 \\
\hline
\end{tabular}

(b)

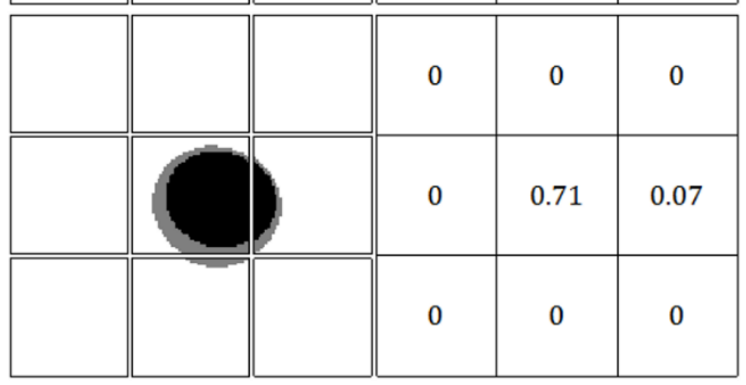

(c)

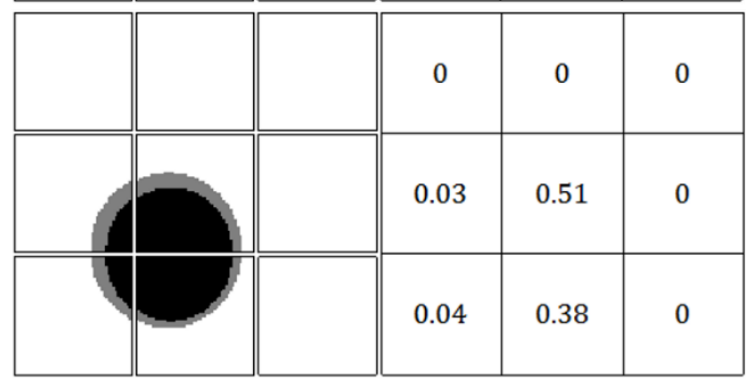

Figure 12. Experimental void geometries for selected cases with black and gray respectively indicating the optically measured small estimate and large estimate of void geometry, (left), with experimentally measured values of $C^{*}$ on the $3 \times 3$ grid of electrode junctions (right). Measurements of $\left|C^{*}\right|<0.02$ are eliminated as noise. Case (c) corresponds to figure 11. 
Sensor for characterization of void content in dielectric layers

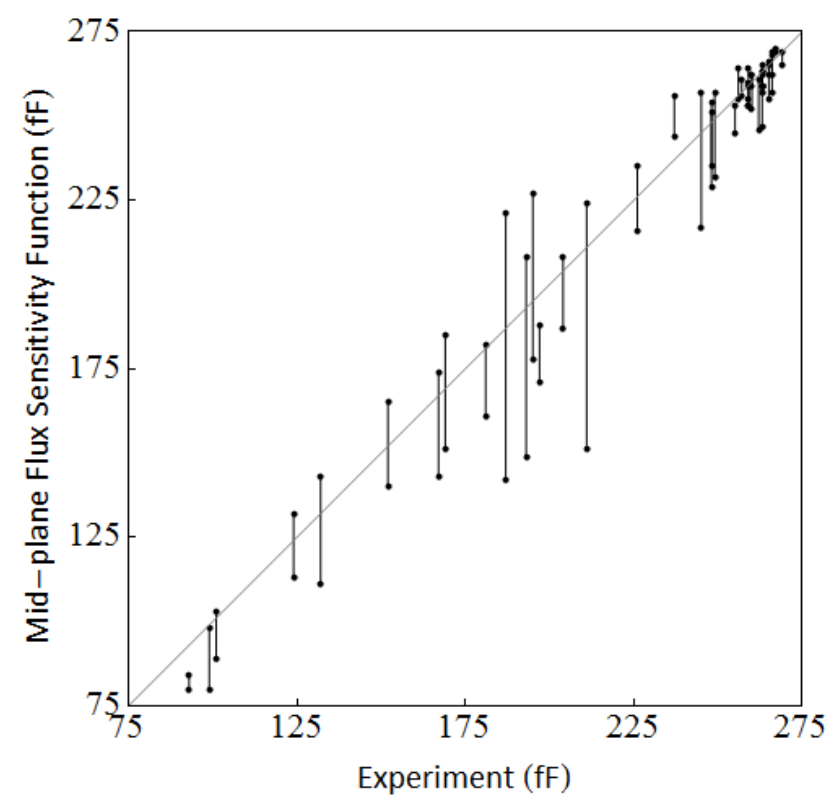

Figure 13. Junction capacitance due to the presence of artificial voids as measured experimentally and predicted using the visualized void boundaries. Each prediction is shown as a span between small and large void boundary limits. 
Sensor for characterization of void content in dielectric layers

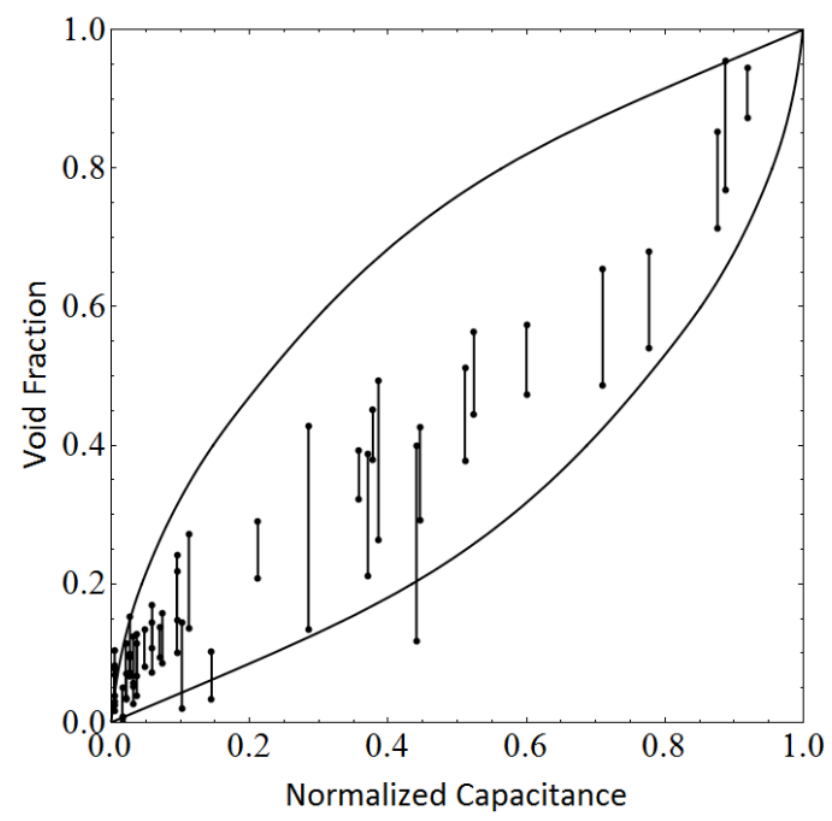

Figure 14. Optically measured void fraction versus normalized capacitance overlaid with void fraction bounds obtained from the mid-plane flux method. 